\title{
Studies on Longer Wavelength Type II Radio Bursts Associated with Flares and CMEs during the Rise and Decay Phase of 23rd Solar Cycle
}

\author{
V. Vasanth and S. Umapathy \\ School of Physics, Madurai Kamaraj University, Madurai 625 021, India \\ Correspondence should be addressed to V. Vasanth; vasanth_velu2007@yahoo.co.in
}

Received 4 December 2013; Revised 13 March 2014; Accepted 2 May 2014; Published 3 June 2014

Academic Editor: Luciano Nicastro

Copyright (C) 2014 V. Vasanth and S. Umapathy. This is an open access article distributed under the Creative Commons Attribution License, which permits unrestricted use, distribution, and reproduction in any medium, provided the original work is properly cited.

A statistical study on the properties of CMEs and flares associated with DH-type II bursts in the 23rd solar cycle during the period 1997-2008 is carried out. A sample of 229 events from our recent work is used for the present study (Vasanth and Umapathy, 2013). The collected events are divided into two groups as (i) solar cycle rise phase events and (ii) solar cycle decay phase events. The properties of CMEs in the two groups were compared and the results are presented. It is noted that there is no difference in the properties of type II burst like start frequency and end frequency between the solar cycle rise phase events and decay phase events. The mean CME speed of solar cycle decay phase events $\left(1373 \mathrm{~km} \mathrm{~s}^{-1}\right)$ is slightly higher than the solar cycle rise phase events $\left(1058 \mathrm{~km} \mathrm{~s}^{-1}\right)$. The mean CME acceleration of solar cycle decay phase events $\left(-15.18 \mathrm{~m} \mathrm{~s}^{-2}\right)$ is found to be higher than that of the solar cycle rise phase events $\left(-1.32 \mathrm{~m} \mathrm{~s}^{-2}\right)$. There exists good correlation between (i) CME speed and width and (ii) CME speed and acceleration for solar cycle decay phase events $(R=0.79, R=-0.80)$ compared to solar cycle rise phase events $(R=0.60$, $R=-0.57)$. These results indicate that the type II bursts parameters do not depend upon the time of appearance in the solar cycle.

\section{Introduction}

Coronal mass ejections (CMEs) are the large eruption of magnetized plasma from sun into heliosphere and are important cause for geomagnetic storms if they are directed towards earth. The shocks driven by the CMEs accelerate electrons and produce type II bursts in the corona and interplanetary medium (IP) [1]. Type II bursts are the longest known signatures of shock wave [2]. In the dynamic spectrum they are observed as slowly drifting (from high to low frequency) emission bands. The first identification of type II bursts was made by Payne-Scott et al. [3] and later Wild and McCready [2] classified them as type II bursts to differentiate them from fast drifting type III bursts. The first observation of type II bursts in the IP medium was made by IMP-6 [4] and Voyager [5] spacecraft missions. Many IP type II bursts are observed by ISEE- 3 spacecraft in the frequency range of $2 \mathrm{MHz}$ to $30 \mathrm{kHz}[6,7]$. The data on DH-type II bursts are provided by radio and plasma wave (WAVES) experiment on board wind spacecraft launched in 1994 [8]. The wind spacecraft observes the type II/IV radio bursts in the frequency range between $14 \mathrm{MHz}$ and $20 \mathrm{kHz}$ by RAD1 and RAD2 due to ionospheric cut-off frequency [9-11]. The frequency range in the decameter hectometric (DH) wavelength domain corresponds to the heights of $2-10 R_{\odot}$ and bridges the gap between the metric type II bursts observed by ground based radio observatories and the kilometric type II bursts observed by space-borne radio instruments.

The CMEs associated with the DH-type II bursts are found to be fast and wide, and they show strong deceleration in the LASCO FOV [12]. It is now well accepted that the type II bursts in $\mathrm{DH}$ and $\mathrm{Km}$ range are driven by CMEs, while the origin of coronal shocks is still under debate [11, 1315]. Lara et al. [16] studied the CMEs associated with type II bursts ( $\mathrm{m}$ - and DH-type II bursts) wavelength range and found that the CMEs associated with metric type II bursts are more energetic than regular CMEs but less energetic than the CMEs associated with DH-type II bursts. The characteristics 
TABLE 1: Properties of DH-type II bursts, flares, and CMEs in solar cycle rise phase and solar cycle decay phase events.

\begin{tabular}{|c|c|c|c|c|}
\hline $\begin{array}{l}\text { Serial } \\
\text { number }\end{array}$ & Properties & $\begin{array}{c}\text { Solar cycle } \\
\text { rise phase events }\end{array}$ & $\begin{array}{c}\text { Solar cycle } \\
\text { decay phase events }\end{array}$ & $\begin{array}{c}t \text {-test } \\
P \text {-value }(\%)\end{array}$ \\
\hline & \multicolumn{4}{|c|}{ DH-type II bursts } \\
\hline 1 & Start frequency $(\mathrm{MHz})$ & $10.65(\mathbf{0 . 3 8})$ & $10.12(\mathbf{0 . 4 7})$ & 37.71 \\
\hline 2 & End frequency $(\mathrm{MHz})$ & $2.48(\mathbf{0 . 2 6})$ & $1.78(\mathbf{0 . 2 5})$ & 5.55 \\
\hline 3 & Duration (min) & $441(67.92)$ & $369(61.09)$ & 43.04 \\
\hline \multirow[t]{2}{*}{4} & Type II speed $\left(\mathrm{km} \mathrm{s}^{-1}\right)$ & 1135 (71.50) & $1260(79.71)$ & 25.51 \\
\hline & \multicolumn{4}{|c|}{ Flares } \\
\hline 5 & Flare duration (min) & $53(4.27)$ & $59(4.35)$ & 33.64 \\
\hline 6 & Flare rise time (min) & $26(\mathbf{1 . 8 9})$ & $31(2.47)$ & 8.84 \\
\hline \multirow[t]{2}{*}{7} & Flare decay time (min) & $27(2.75)$ & $28(2.67)$ & 84.99 \\
\hline & \multicolumn{4}{|c|}{ CMEs } \\
\hline 8 & CME speed $\left(\mathrm{km} \mathrm{s}^{-1}\right)$ & $1058(47.53)$ & 1377 (58.02) & $\ll 1$ \\
\hline 9 & Width (deg) & $260(\mathbf{1 0 . 4 2 )}$ & $276(\mathbf{1 0 . 5 9 )}$ & 26.59 \\
\hline 10 & Acceleration $\left(\mathrm{m} \mathrm{s}^{-2}\right)$ & $-1.32(2.29)$ & $-15.18(4.45)$ & 2.01 \\
\hline
\end{tabular}

${ }^{*}$ The mean (standard error) values of the properties are given in this table.

of CMEs associated with DH-type II bursts are studied by several authors [11, 12, 17-19]. The DH-type II bursts are found to be associated with strongly energetic and wide CMEs, and these CMEs are good indicators of geomagnetic storms at earth if they appear on the front side of the solar disk [12].

In the present work, we aimed to study the properties of CMEs and flares associated with DH-type II bursts in the rise and decay phase of 23rd solar cycle during the period 19972008. The method of data analysis is described in Section 2, the results are presented in Section 3, and the conclusions are discussed in Section 4.

\section{Data Analysis}

A set of $344 \mathrm{DH}$-type II bursts associated with flares and CMEs listed in the Wind/WAVES type II catalog (http:// cdaw.gsfc.nasa.gov/CME_list/radio/waves_type2.html) in the 23rd solar cycle during the period 1997-2008 are used in the present analysis. The characteristics of associated CME data such as speed, width, and acceleration are obtained from the LASCO CME catalog (http://cdaw.gsfc.nasa.gov/CME_list) maintained by the CDAW data center [20,21]. CME can be directly observed from 2 to $32 R_{\odot}$ from the sun using the SOHO/LASCO C2 and C3 coronagraphs [22]. The properties of CMEs listed in the LASCO CME catalog are used for the analysis without correcting the projection effects. We have used the following selection criteria to select the events for our further analysis. They are as follows.

(i) The source location of the events should be clear.

(ii) The flare importance should be clear.

(iii) Backside events should be excluded.

Using the above selection criteria, 232 events are selected out of 344 events in the type II catalog. Further out of 232 events, 3 complex events are removed from the present study therefore the total number of events reduces to 229 events.
Each event contains DH-type II bursts and its associated flares and CMEs. The 23rd solar cycle covering the period 1997-2008 has double peaked solar maximum. The first peak occurred during April, 2000 and the second peak during April, 2002. Therefore, we classified our events into groups based on the behavior of solar cycle as

(i) solar cycle rise phase events (DH-type II bursts associated with flares and CMEs that are observed during the period April 1997-April 2002);

(ii) solar cycle decay phase events (DH-type II bursts associated flares and CMEs that are observed during the period May 2002-April 2008).

Out of 229 events, 127 events occurred during the solar cycle rise phase and the remaining 102 events occurred in the solar cycle decay phase of the 23rd solar cycle during the period 1997-2008. The properties of type II bursts, flares, and CMEs in the solar cycle rise phase and in solar cycle decay phase were compared and the results are discussed in the next section.

\section{Results and Discussions}

The statistical properties of DH-type II bursts, flares, and CMEs in the solar cycle rise and decay phase are investigated and the results are summarized in Table 1 . In this table, column 1 specifies the characteristic properties; column 2 defines the statistical properties. The statistical properties of solar cycle rise phase events and those for solar cycle decay phase events are given in column 3 and column 4 , respectively. The $t$-test $P$ value specifies the statistical significance of the mean values between the two groups and they are given in column 5 .

3.1. Properties of Type II Bursts. The distributions of start frequency of the DH-type II bursts in the solar cycle rise 


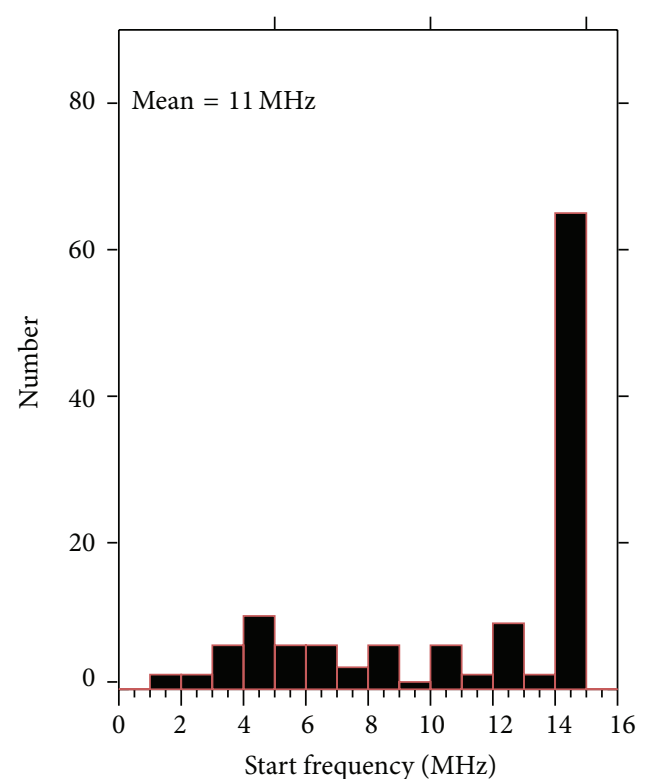

(a)

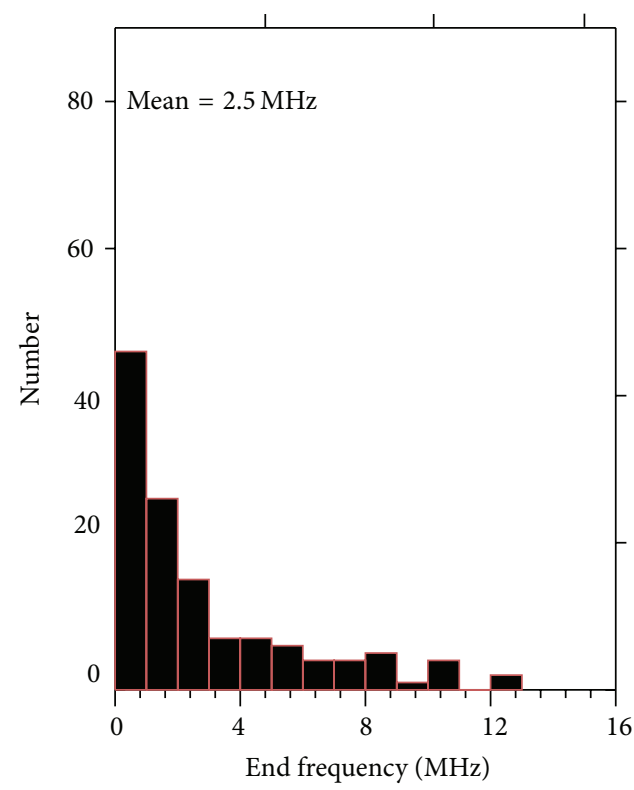

(c)

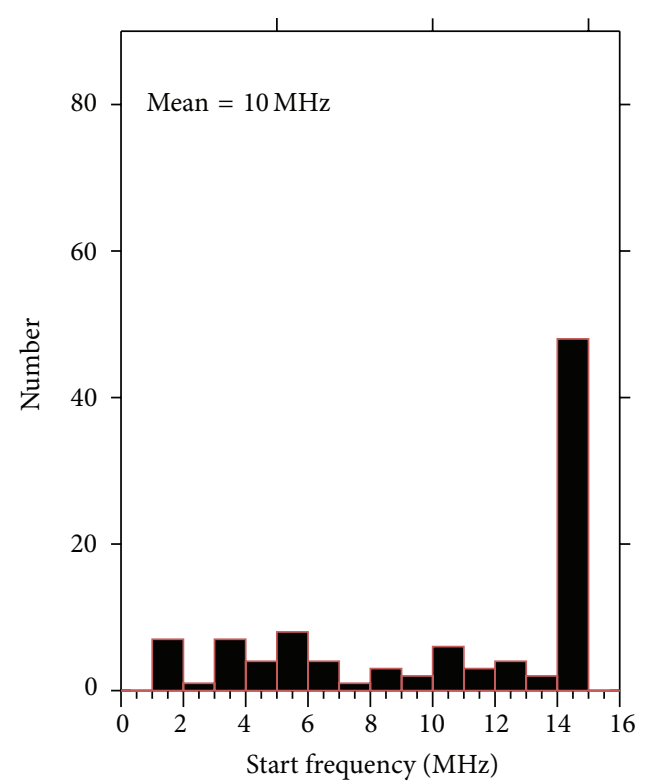

(b)

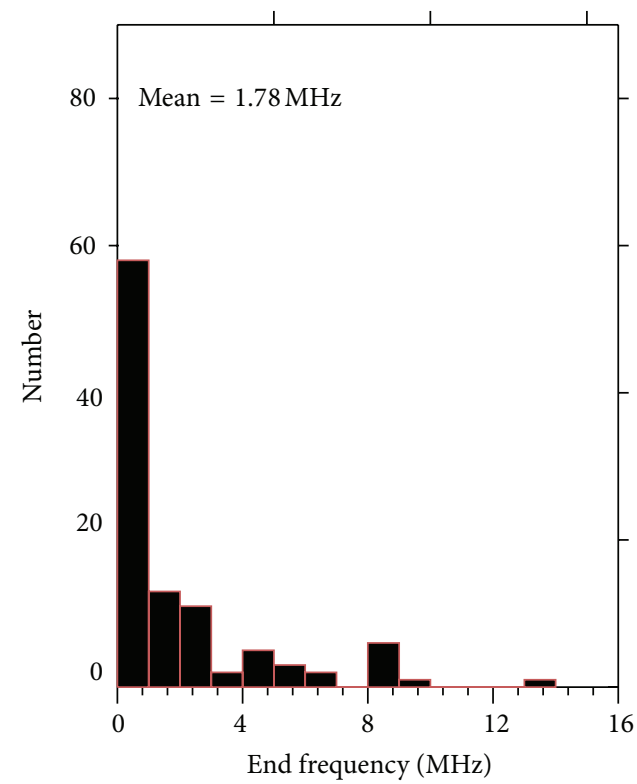

(d)

Figure 1: Distribution of start frequency of DH-type II bursts for (a) solar cycle rise phase events and (b) solar cycle decay phase events. Similarly the distribution of end frequency of DH-type II bursts for (c) solar cycle rise phase events and (d) solar cycle decay phase events.

and decay phase events are shown in Figures 1(a) and 1(b), respectively. The start frequency of most of the DH-type II bursts varies between $14 \mathrm{MHz}$ and $1 \mathrm{MHz}$. In both groups, about $50 \%$ of the DH-type II bursts start at $14 \mathrm{MHz}$ and are the upper cut-off frequency of the WAVES instrument, which indicates that there is a chance for some of the events to be continuation of m-type II bursts. DH-type II bursts are radio signatures of CME driven shocks [12]. The start frequency indicates the height at which the radio emission from the shocks becomes visible [23]. The mean start frequency of type II bursts in solar cycle rise phase events is $11 \mathrm{MHz}$, whereas for the solar cycle decay phase events it is $10 \mathrm{MHz}$ as seen in Table 1 . The difference between the means is not statistically significant with $t$-test $P$ value $>5 \%$ as seen in Table 1 .

The distributions of end frequency of the DH-type II bursts in the solar cycle rise and decay phase events are shown in Figures 1(c) and 1(d), respectively. The end frequency varies between $12 \mathrm{MHz}$ and $20 \mathrm{kHz}$. The end frequency is indicative of CME energies [23, 24]. The shocks produced by fast and wide CMEs travel far into the interplanetary medium. In solar cycle rise phase $50 \%$ of the type II events and in solar cycle decay phase $61 \%$ of the type II events end 


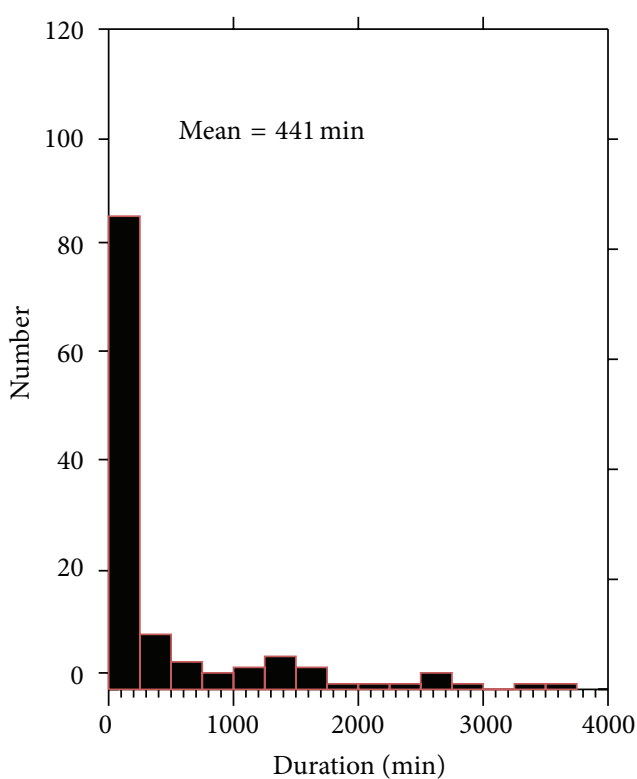

(a)

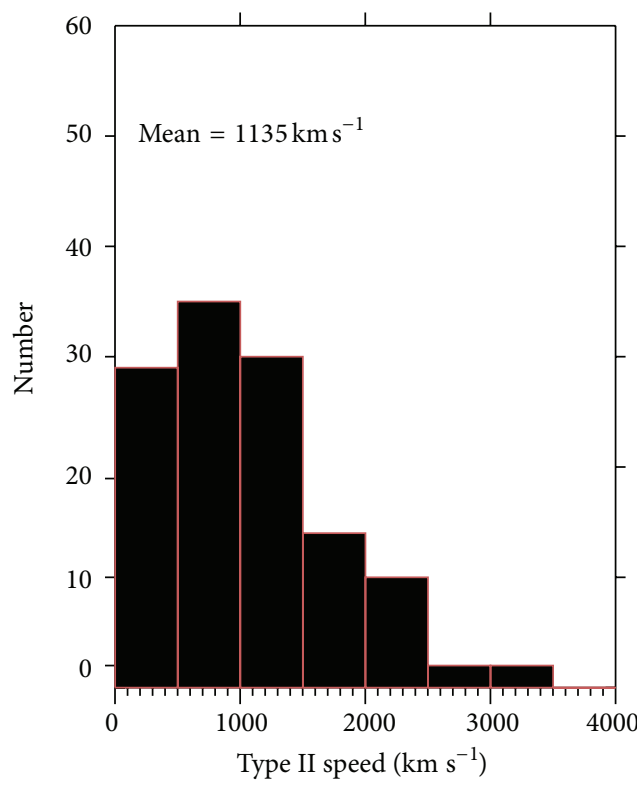

(c)

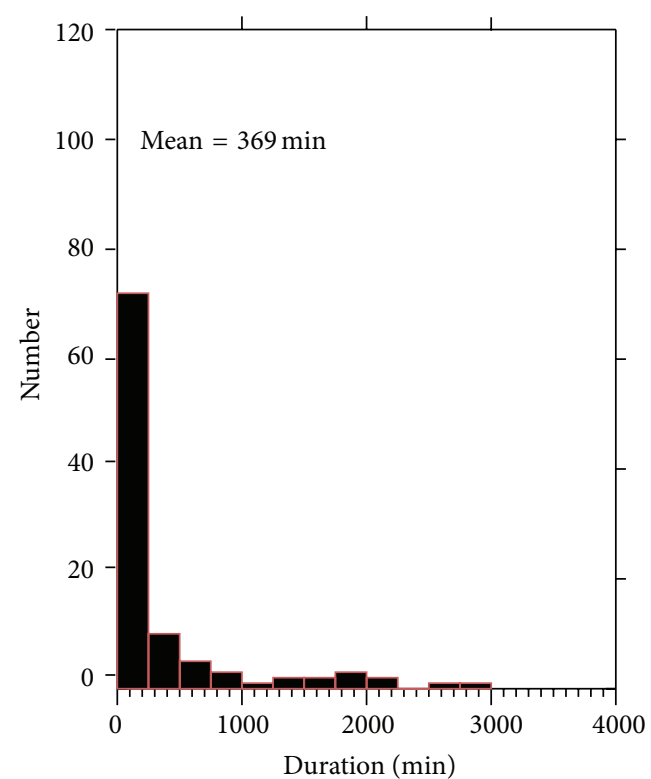

(b)

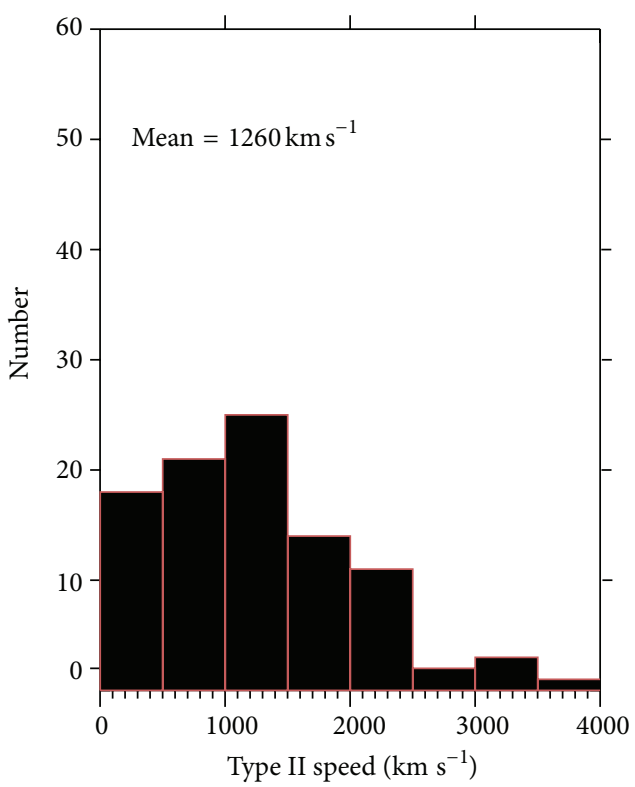

(d)

Figure 2: Distributions of duration of DH-type II bursts for (a) solar cycle rise phase events and (b) solar cycle decay phase events and shock speed of DH-type II bursts for (c) solar cycle rise phase events and (d) solar cycle decay phase events.

below $1 \mathrm{MHz}$. Majority of the type II events in both groups have end frequency around/below $1 \mathrm{MHz}$ which indicates that they continue into km range (shown by first bin of Figures 1(c) and $1(\mathrm{~d})$, resp.). The mean end frequency of solar cycle rise phase events is $3 \mathrm{MHz}$ and that of the solar cycle decay phase events is $2 \mathrm{MHz}$ as seen in Table 1 . The difference between the means is less statistically significant with $t$-test $P$ value $=6 \%$ as seen in Table 1.

Figures 2(a) and 2(b) show the distributions of duration of the DH-type II bursts in solar cycle rise and in decay phase events. The duration of DH-type II bursts varies between few minutes to long time as 4000 minutes. In solar cycle rise phase $68 \%$ and in solar cycle decay phase $70 \%$ of the DH-type II bursts duration lie within $200 \mathrm{~min}$. The mean duration of DH-type II bursts in solar cycle rise phase events is $441 \mathrm{~min}$ and that of the solar cycle decay phase events is $369 \mathrm{~min}$ as seen in Table 1. The difference between the means is not statistically significant with $t$-test $P$ value $>5 \%$ as seen in Table 1.

The shock speed of the DH-type II bursts was estimated by applying Leblanc electron density model $[18,25]$. The model is valid for $R>1.2 R_{\odot}$ and not for very low heights. 


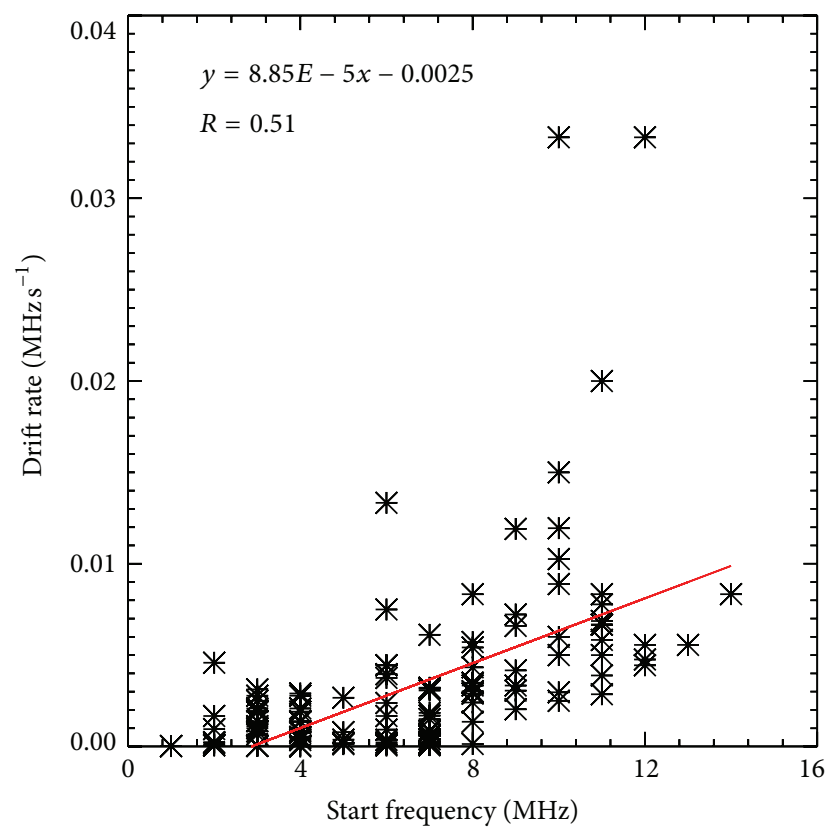

(a)

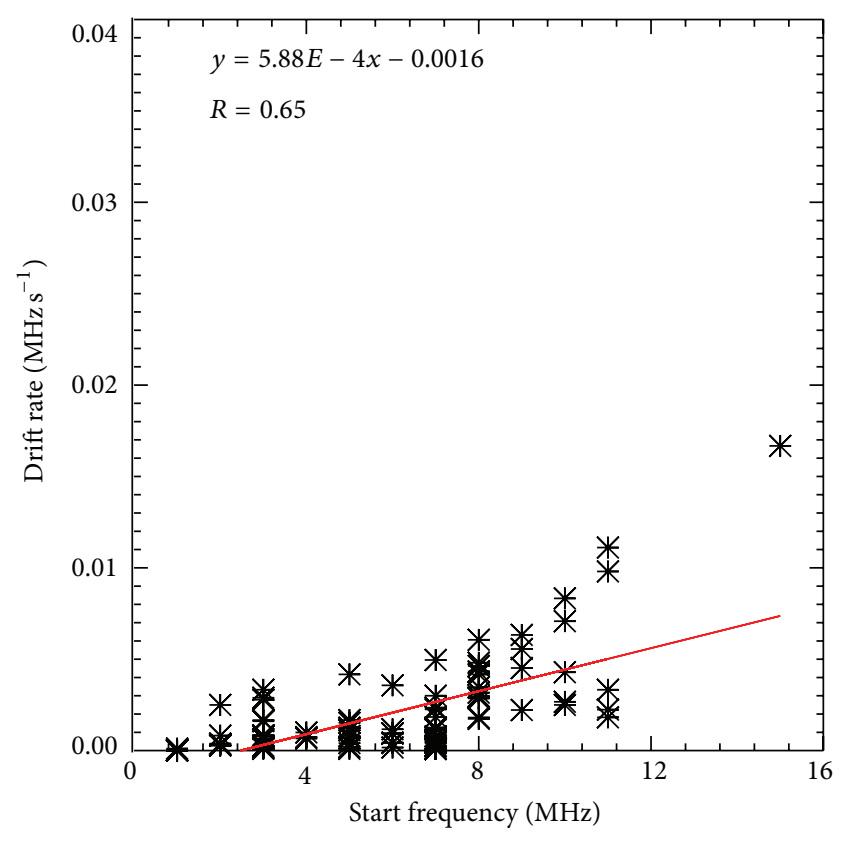

(b)

FIGURE 3: Correlation between start frequency and drift rate for (a) solar cycle rise phase events and (b) solar cycle decay phase events.

For each measured frequency, their corresponding electron density number and radial distance are obtained using the Leblanc electron density model from which the shock speed of the DH-type II bursts is estimated. The distributions of shock speeds inferred from the DH-type II burst drift rates for the solar cycle rise and decay phase events are presented in Figures 2(c) and 2(d), respectively. The shock speed varies between few hundred $\mathrm{km} \mathrm{s}^{-1}$ and $4000 \mathrm{~km} \mathrm{~s}^{-1}$. The mean shock speed of DH-type II bursts in solar cycle rise phase events is $1135 \mathrm{~km} \mathrm{~s}^{-1}$ and that of the solar cycle decay phase events is $1260 \mathrm{~km} \mathrm{~s}^{-1}$ as seen in Table 1 . The difference between the means is not statistically significant with $t$-test $P$ value $>5 \%$ as seen in Table 1 .

The correlations between mean starting frequency of $\mathrm{DH}$ type II bursts and drift rate for solar cycle rise and decay phase events are shown in Figures 3(a) and 3(b), respectively. Figure 3 shows that there might be dependence between the mean starting frequency and drift rate for both groups of events. The correlation is better for solar cycle decay phase events as compared to solar cycle rise phase events $(R=0.65$ and $R=0.51$, resp.). It is clear from the figure that the drift rate increases with start frequency [26].

3.2. Properties of Associated Flares. The distributions of flare rise time associated with DH-type II bursts in the solar cycle rise and decay phase events are shown in Figures 4(a) and 4(b), respectively. The flare rise time (the time difference between flare start and flare peak) helps in establishing the relationship between flares and type II bursts [11, 27], since type II burst starting between flare start and flare peak has a chance to be driven by flare shocks. Among solar cycle rise phase events 54\% (69/127) of the flares rise within $20 \mathrm{~min}$ and the remaining $46 \%$ (58/127) of the events have longer flare rise time, while among solar cycle decay phase events only $39 \%$ (40/102) of the flares rise within $20 \mathrm{~min}$ and the remaining $61 \%(62 / 102)$ have longer flare rise time. The mean flare rise time in solar cycle rise phase events is $26 \mathrm{~min}$ and that of the solar cycle decay phase events is $31 \mathrm{~min}$ as seen in Table 1 . The difference between the means is not statistically significant with $t$-test $P$ value $>5 \%$ as seen in Table 1 .

The distributions of flare decay time associated with $\mathrm{DH}-$ type II bursts in the solar cycle rise and decay phase events are shown in Figures 4(c) and 4(d), respectively. The flare decay time (the time difference between flare peak and flare end) varies between few minutes and $200 \mathrm{~min}$. Among solar cycle rise phase events 55\% (70/127) and among solar cycle decay phase events $56 \%(57 / 102)$ of the flares decay within $20 \mathrm{~min}$. The mean flare decay time in solar cycle rise phase events is $27 \mathrm{~min}$ and that of the solar cycle decay phase events is $28 \mathrm{~min}$. The difference between the means is less statistically significant with $t$-test $P$ value $=9 \%$ as seen in Table 1 .

Figures 5(a) and 5(b) show the distribution of flare duration associated with DH-type II bursts in the solar cycle rise and decay phase events. Among solar cycle rise phase events, $61 \%(78 / 127)$ have duration within $50 \mathrm{~min}$, while among solar cycle decay phase events, only 55\% (56/102) of the events have duration within $50 \mathrm{~min}$. The mean flare duration in solar cycle rise phase events is $53 \mathrm{~min}$ and that of the solar cycle decay phase events is $59 \mathrm{~min}$. The longer flare duration indicates the enhanced action of Lorentz force and the associated CMEs may have higher speed and acceleration 


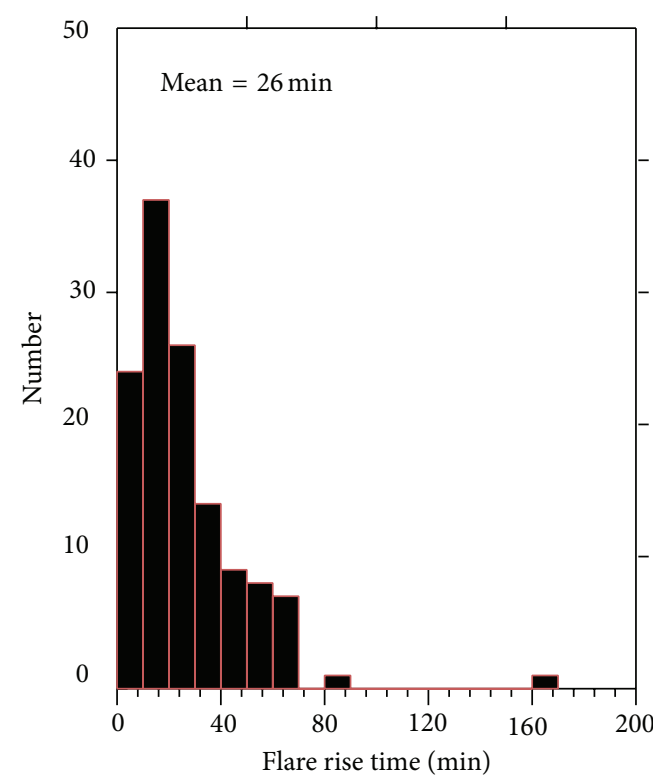

(a)

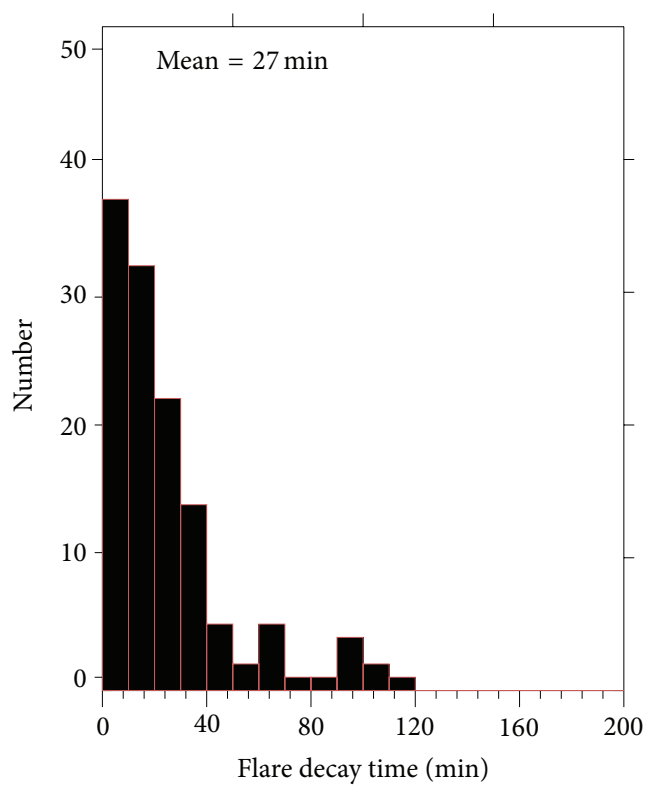

(c)

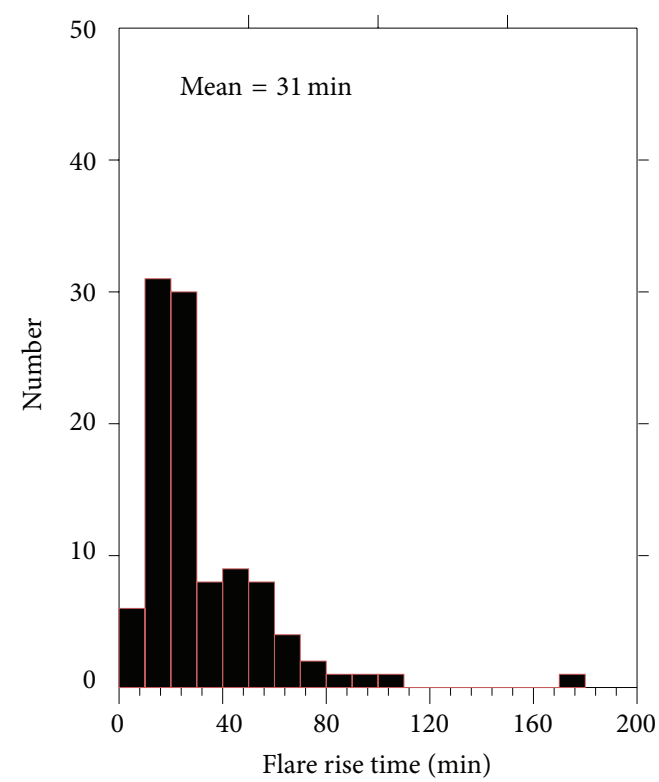

(b)

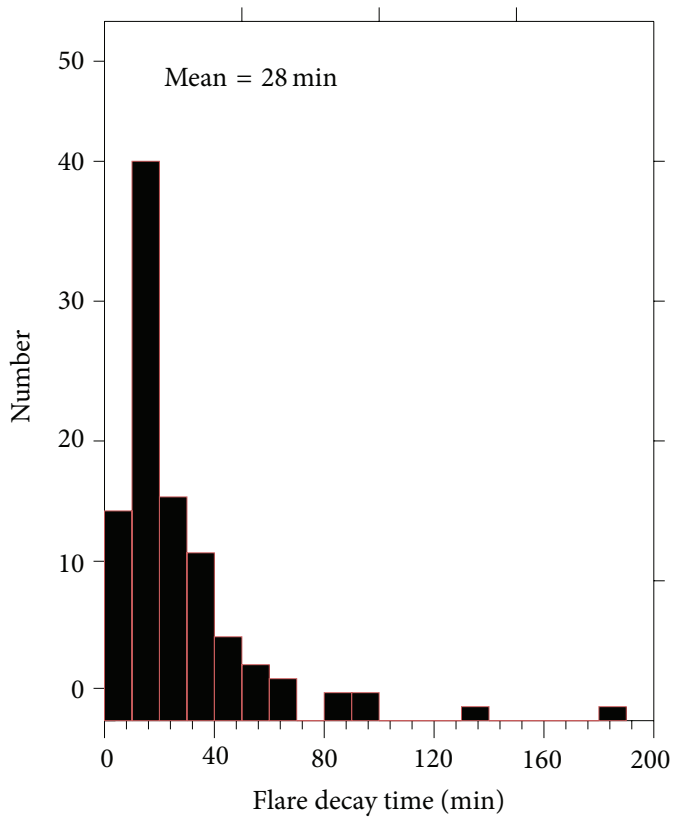

(d)

FIGURE 4: Distributions of flare rise time for (a) solar cycle rise phase events and (b) solar cycle decay phase events and flare decay time for (c) solar cycle rise phase events and (d) solar cycle decay phase events.

[28]. The difference between the means is not statistically significant with $t$-test $P$ value $>5 \%$ as seen in Table 1 .

The distributions of flare classes associated with CMEs and DH-type II bursts for the solar cycle rise and decay phase events are shown in Figures 5(c) and 5(d), respectively. The $\mathrm{X}$-ray flare class is classified as $\mathrm{A}, \mathrm{B}, \mathrm{C}, \mathrm{M}$, and $\mathrm{X}$ depending on their intensities, where $\left(A=10^{-8}, B=10^{-7}, C=10^{-6}, M=\right.$ $10^{-5}$, and $\mathrm{X}=10^{-4} \mathrm{Wm}^{-2}$ ) the value after the flare class is the multiplication factor. For example, M3.4 has a flux of $3.4 \times$ $10^{-5} \mathrm{Wm}^{-2}$. In solar cycle rise phase events, 25\% (32/127) are
X-class flares, 52\% (66/127) are M-class flares, 19\% (24/127) are C-class flares, and 4\% (5/127) are B-class flares, while in solar cycle decay phase events 30\% (31/102) are X-class flares, 47\% (48/102) are M-class flares, 22\% (22/102) are Cclass flares, and 1\% (1/102) are B-class flares. The solar cycle decay phase events are associated with more energetic X-class flares than solar cycle rise phase events. We expect that the stronger flares will be associated with fast CMEs [29, 30].

The distributions of source location of flares associated with DH-type II bursts for the solar cycle rise phase and decay 


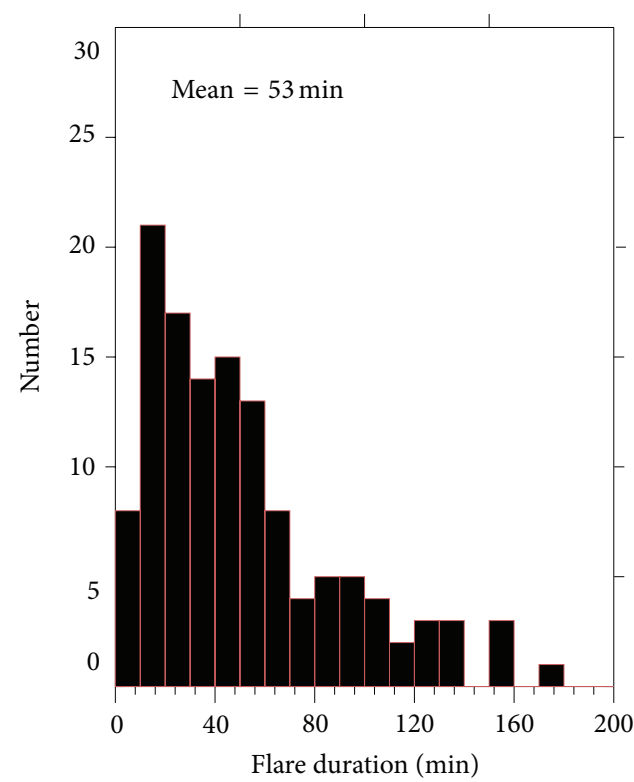

(a)

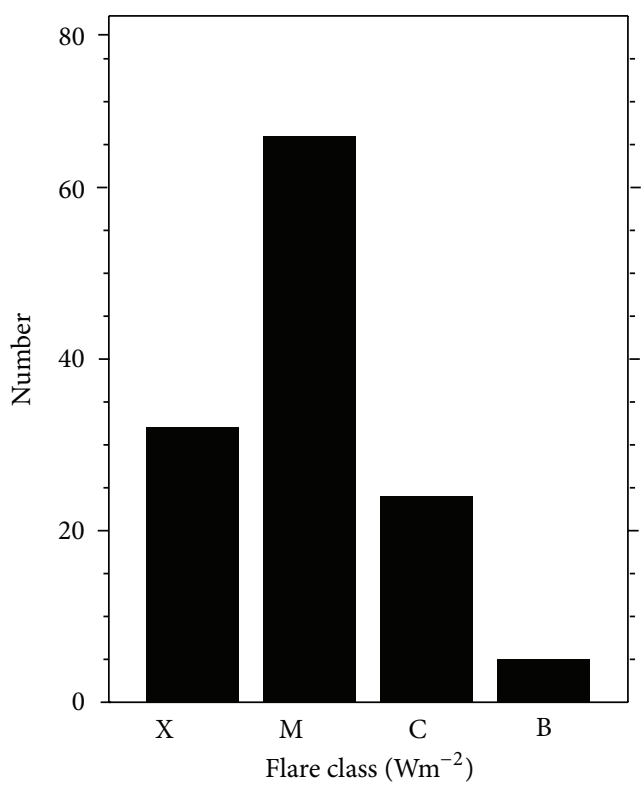

(c)

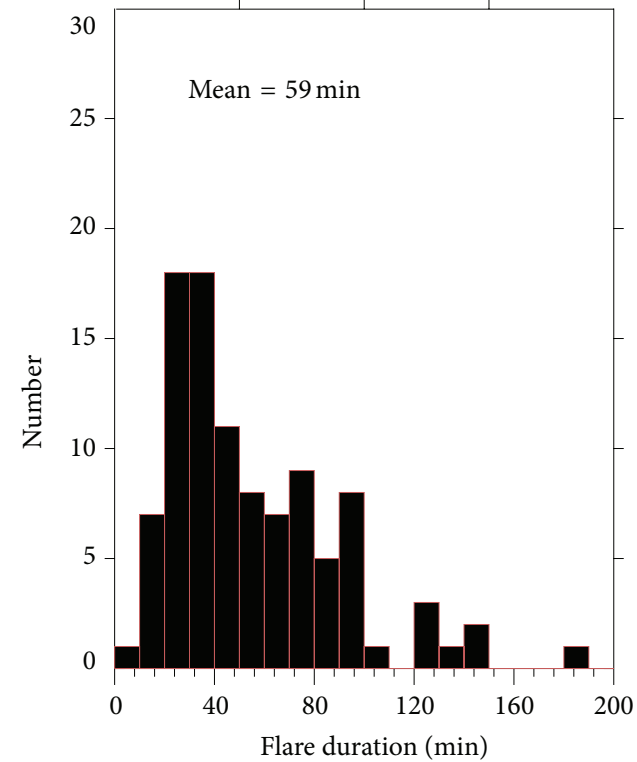

(b)

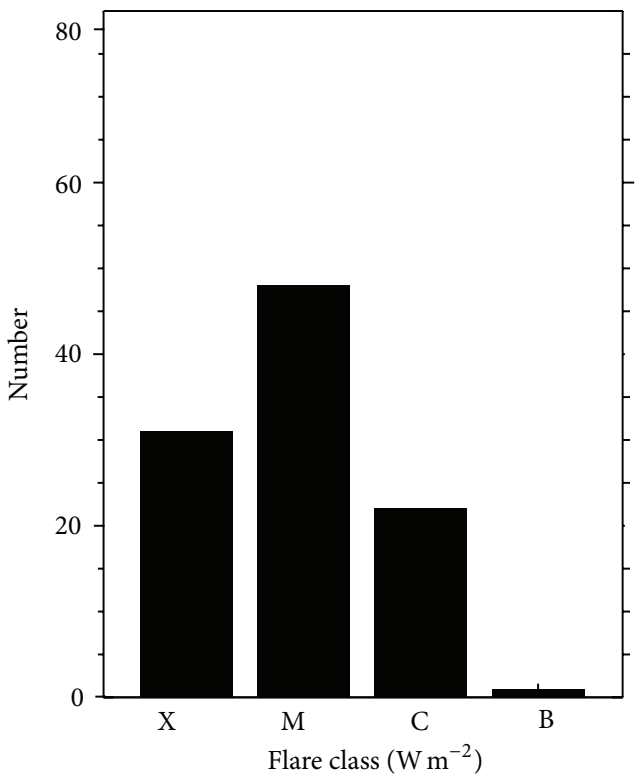

(d)

FIGURE 5: Distributions of flare duration for (a) rise phase events and (b) decay phase events and flare classes for (c) rise phase events and (d) decay phase events.

phase events are shown in Figures 6(a) and 6(b), respectively. Among solar cycle rise phase events, 64\% (81/127) originate in western hemisphere and the remaining 36\% (46/127) originate in eastern hemisphere. Similar result was found for solar cycle decay phase events; 60\% (61/102) originate in western hemisphere and the remaining $40 \%$ (41/102) originate in eastern hemisphere. It is clear that majority of the events $62 \%(142 / 229)$ in both cases originate in the western hemisphere.

3.3. Properties of Associated CMEs. The properties of CMEs (like speed, width, acceleration, etc.) listed in the LASCO
CME catalog are used in the present study. All the parameters measured in the plane of the sky will suffer from projection effects and no attempt has been made to correct the projection effects. The properties of CMEs in solar cycle rise and decay phase events are compared and the results are discussed.

The distributions of CME speed associated with flares and DH-type II bursts for the solar cycle rise and decay phase events are shown in Figures 6(c) and 6(d), respectively. The CME speeds are measured from the height-time measurements projected in the plane of the sky. The speed of CMEs varies between few tens of $\mathrm{km} \mathrm{s}^{-1}$ and $3000 \mathrm{~km} \mathrm{~s}^{-1}$. We found 


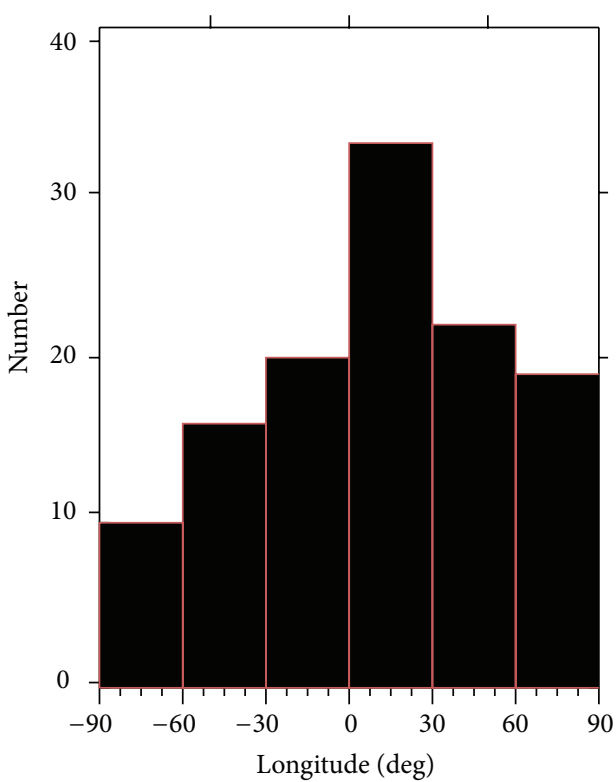

(a)

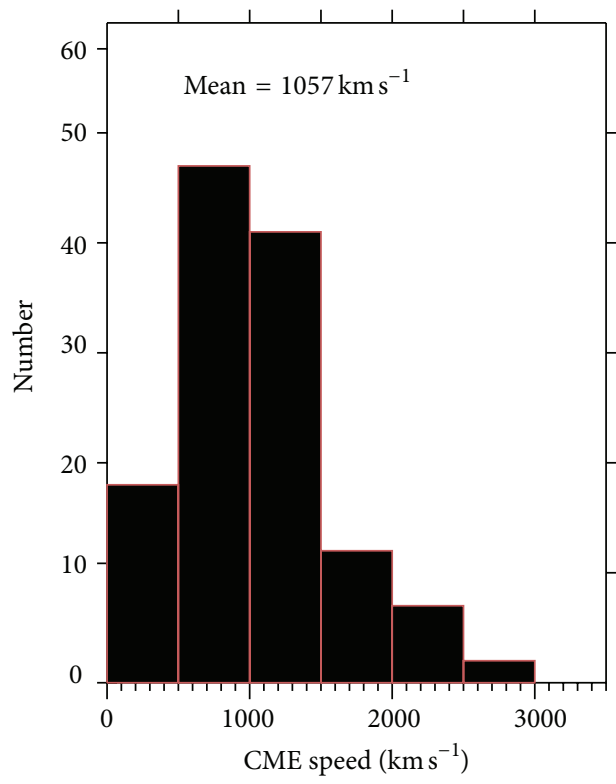

(c)

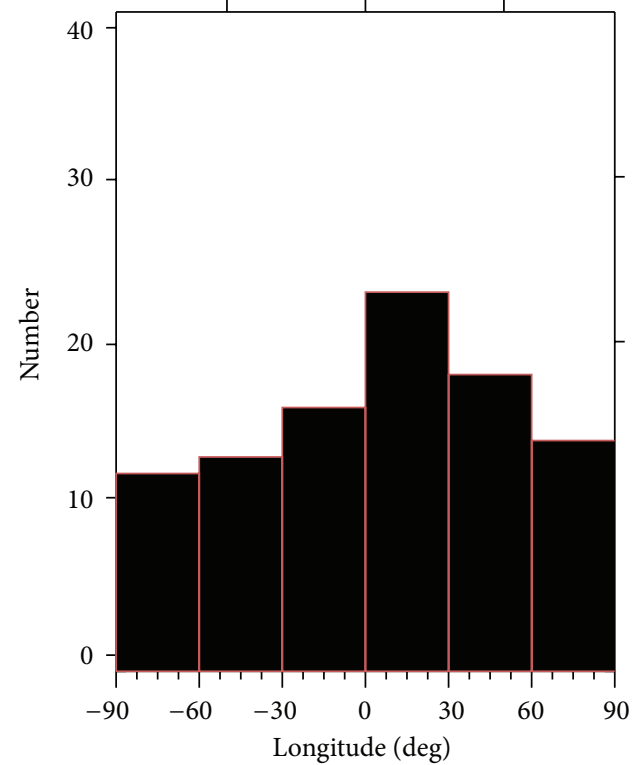

(b)

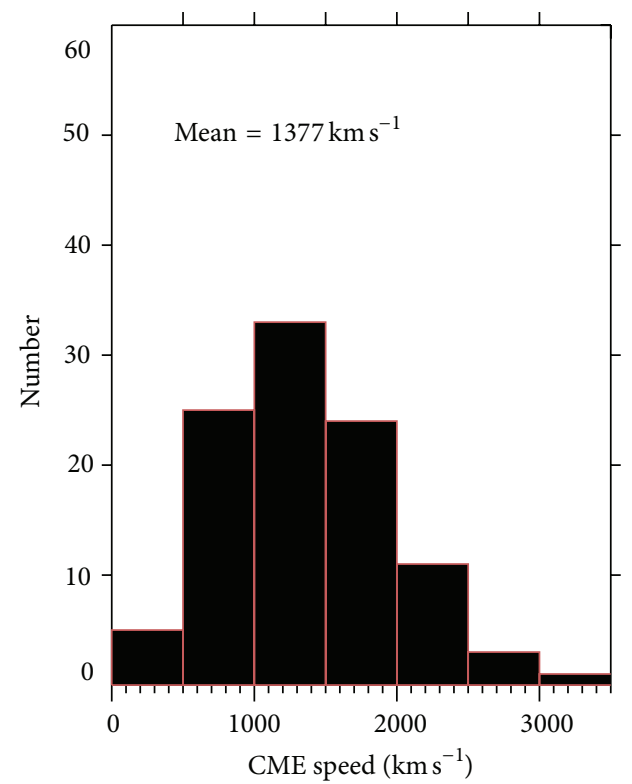

(d)

FIGURE 6: Distributions of longitude for (a) solar cycle rise phase events and (b) solar cycle decay phase events and apparent speed of CMEs for (c) solar cycle rise phase events and (d) solar cycle decay phase events.

that the mean speed of CMEs in solar cycle rise phase events amounts about $1058 \mathrm{~km} \mathrm{~s}^{-1}$. Somewhat higher mean speed of CMEs $\left(1373 \mathrm{~km} \mathrm{~s}^{-1}\right)$ was found for the solar cycle decay phase events. Therefore, the solar cycle decay phase events are found to have higher CME speed compared to solar cycle rise phase events. The difference between the means is statistically significant with $t$-test $P$ value $<1 \%$ as seen in Table 1 .

The distributions of projected width of CMEs associated with flares and DH-type II bursts for the solar cycle rise and decay phase events are shown in Figures 7(a) and 7(b), respectively. The width of the CMEs was estimated in the LASCO C2 FOV and listed in the catalog are used for the analysis. The angular width is measured as the angular extent between the two edge position angles in the sky plane. The width of CMEs varies between $30^{\circ}$ and $360^{\circ}$. We did not find significant difference in the mean width of CMEs in solar cycle rise phase events $\left(260^{\circ}\right)$ and in mean width of CMEs in solar cycle decay phase events $\left(275^{\circ}\right)$. In solar cycle rise phase events, 54\% (70/130) are halo CMEs and in solar cycle decay phase events 58\% (59/102) are halo CMEs. The difference 


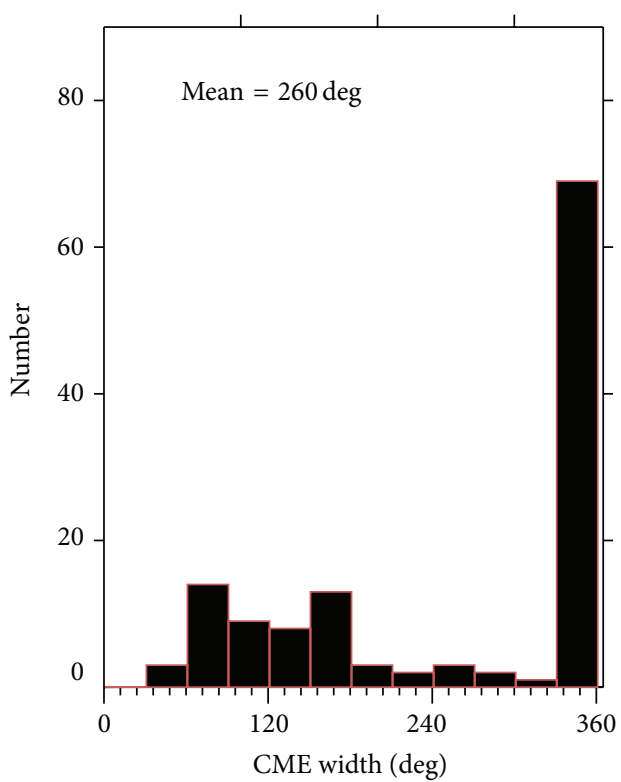

(a)

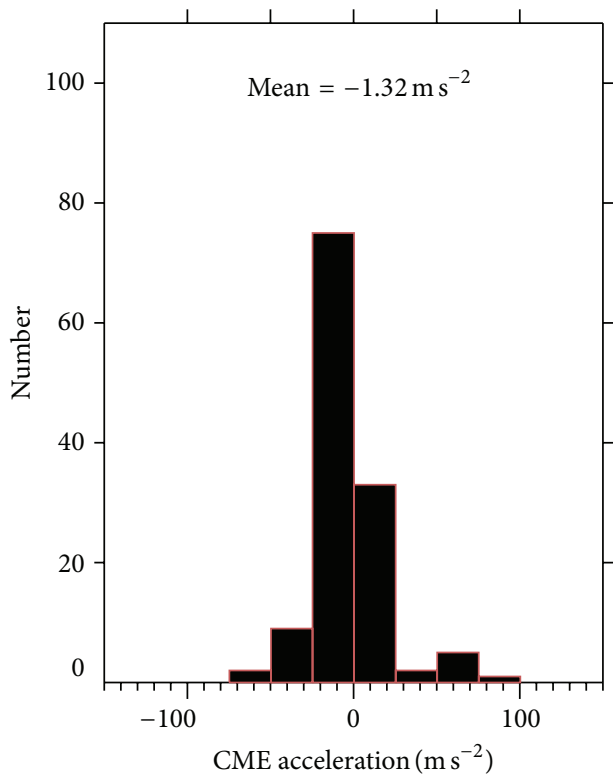

(c)

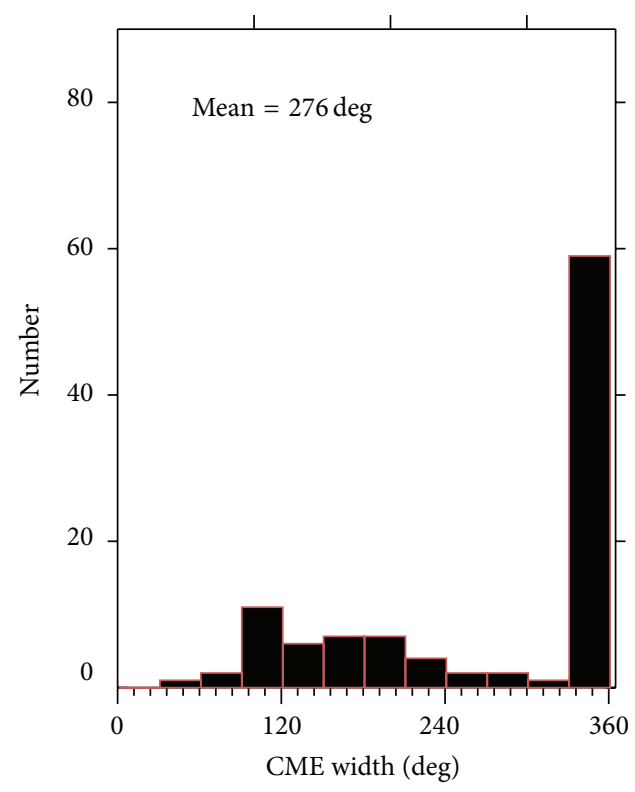

(b)

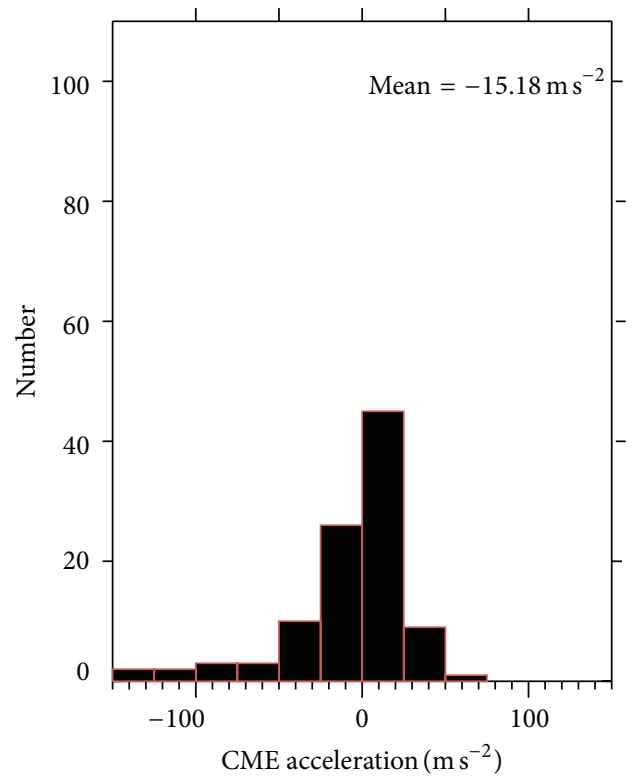

(d)

FIGURE 7: Distributions of apparent width of CMEs for (a) solar cycle rise phase events and (b) solar cycle decay phase events and acceleration of CMEs for (c) solar cycle rise phase events and (d) solar cycle decay phase events.

between the means is not statistically significant with $t$-test $P$ value $>5 \%$ as seen in Table 1 .

The distributions of acceleration of CMEs associated with flares and DH-type II bursts in the solar cycle rise and decay phase events are shown in Figures 7(c) and 7(d), respectively. The CMEs with data point $\geq 4$ are used for the further analysis, so that the acceleration is reliable. Therefore, the acceleration of CMEs is estimated in the full LASCO FOV. The CMEs will accelerate/decelerate depending on their interaction with the solar wind in the LASCO FOV [19]. The fast CMEs decelerate, while the slow CMEs accelerate in the LASCO FOV [21].
The acceleration and deceleration of CMEs depend on the Lorentz force, drag force, and gravity force. The Lorentz force acts longer in accelerating CMEs and shorter in decelerating CMEs; thereby the drag force dominates the dynamics of CMEs with decrease in Lorentz and gravity force and the CME decelerates in the entire LASCO FOV $[18,19]$. In Figure 7, the acceleration of CMEs in the rise phase events ranges between $-80 \mathrm{~m} \mathrm{~s}^{-2}$ and $100 \mathrm{~m} \mathrm{~s}^{-2}$, while in the solar cycle decay phase events it ranges between $-180 \mathrm{~m} \mathrm{~s}^{-2}$ and $60 \mathrm{~m} \mathrm{~s}^{-2}$. This shows that the faster CMEs in the solar cycle decay phase decelerate more than those in the solar cycle 


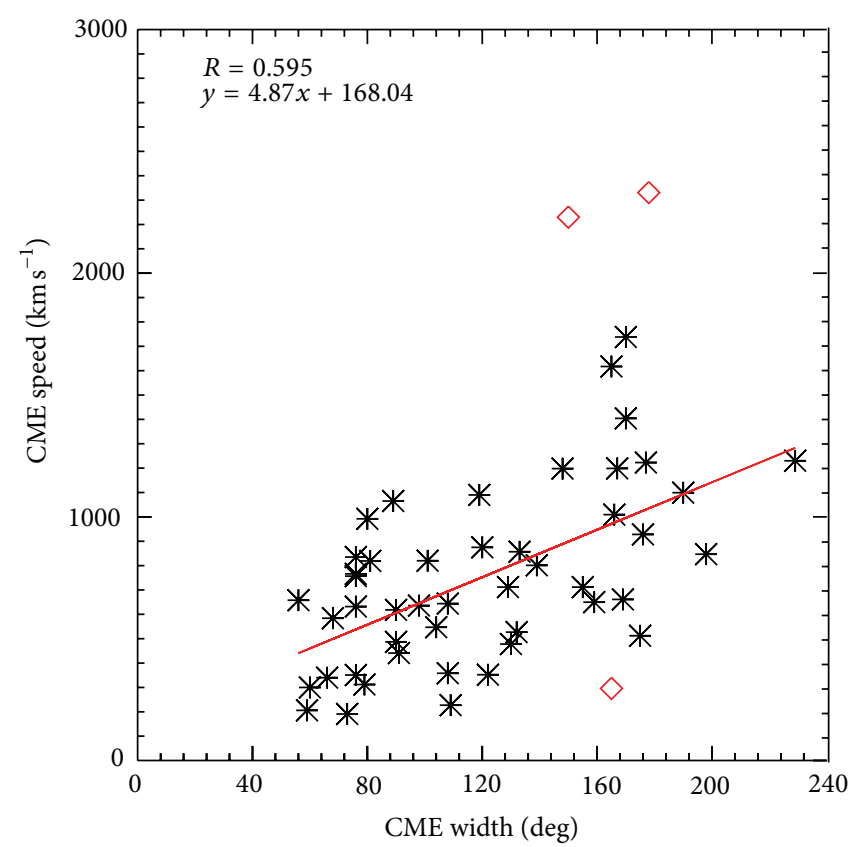

(a)

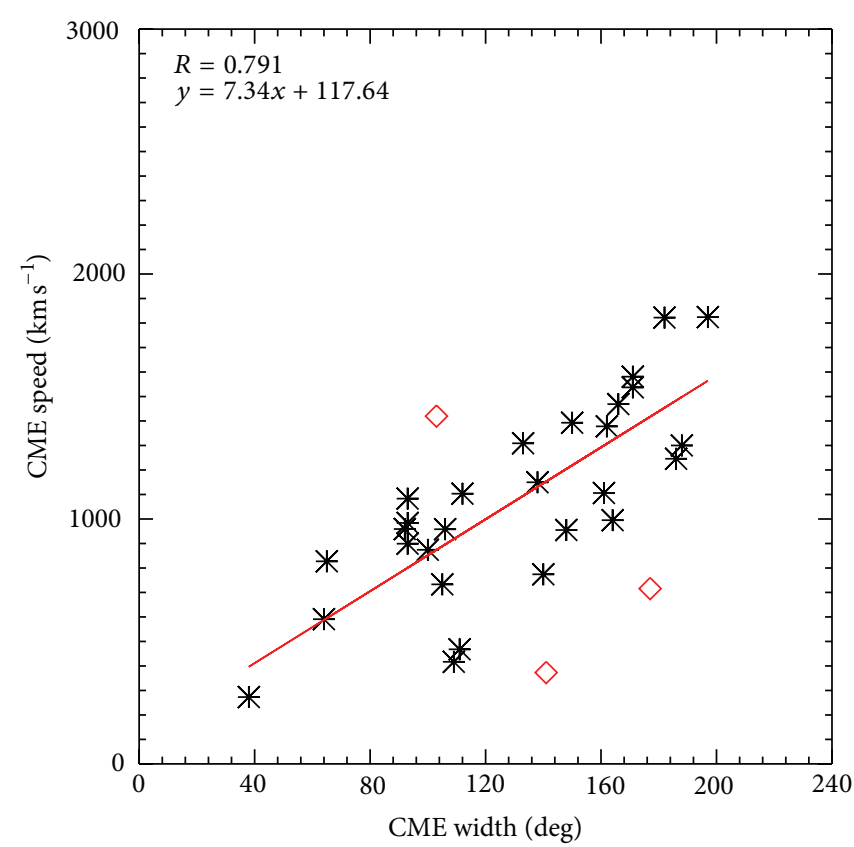

(b)

Figure 8: Correlation between projected speed and width of CMEs for (a) rise phase events and (b) decay phase events (red diamonds are outlier points).

rise phase events. The mean acceleration of 99 solar cycle rise phase events is $-1.32 \mathrm{~m} \mathrm{~s}^{-2}$ and that of the 72 solar cycle decay phase events is $-15.18 \mathrm{~m} \mathrm{~s}^{-2}$. The difference between the means is statistically significant with $t$-test $P$ value $=2.01 \%$ as seen in Table 1.

The correlation between projected speed and width of CMEs for solar cycle rise phase and decay phase events is shown in Figure 8. It is difficult to find the true width of halo and partial halo CMEs. Therefore, we have excluded the full halo CMEs and wider CMEs with width $\geq 200^{\circ}$ from the present analysis. There exists correlation for solar cycle decay phase events with a correlation coefficient of $R=0.79$ and solar cycle decay phase events with $R=0.60$ with the removal of outliers (the outliers are the extreme/error in the data points which affects the correlation, so they are removed from the analysis) shown by red diamond in Figure 8, and these results are in agreement with the earlier reports $[12,18,19]$.

The correlation between speed and acceleration of CMEs for solar cycle rise and decay phase events is shown in Figure 9. Out of 229 events, 171 events are having 4 data points and are considered for the analysis. Out of 171 events, 105 events (61\%) are decelerating and the remaining 66 events (39\%) accelerate in the LASCO FOV. In 127 solar cycle rise phase events, only 99 events are found to have 4 data points, $59 \%(58 / 99)$ are decelerating events, and the remaining $41 \%$ (41/99) are accelerating events, while, in 102 solar cycle decay phase events, only 72 events are found to have 4 data points, $65 \%$ (47/72) are decelerating events, and the remaining 35\% $(25 / 72)$ are accelerating events. The faster events decelerate more with negative correlation between speed and acceleration of CMEs. There exists a good correlation for solar cycle decay phase events with a correlation coefficient of $R=-0.80$, while a weak correlation with $R=-0.57$ exists for solar cycle rise phase events with the removal of outliers (the outliers are the extreme/error in the data points which affects the correlation, so they are removed from the analysis) shown by red diamond in Figure 9. The acceleration decreases with increase in speed of CMEs. The faster CMEs decelerate more than slower CMEs, because of the drag force faced by the CMEs in the interplanetary medium $[12,18,19,21,31]$. Earlier reports $[12,21]$ suggest that the CMEs faster than $900 \mathrm{~km} \mathrm{~s}^{-1}$ have tendency to decelerate in the LASCO FOV.

The correlation between CME speed and DH-type II end frequency for solar cycle rise and decay phase events is shown in Figure 10. The correlations between ending frequency and CME speed are very weak for both data sets $(R=-0.43$ and $R=-0.36$ for solar cycle rise and decay phase events, resp.).

\section{Conclusions}

The properties of flares and CMEs associated with DH-type II bursts during the period 1997-2008 in the 23rd solar cycle are investigated. The collected events are divided into two groups as (i) solar cycle rise phase events and (ii) solar cycle decay phase events. The main results of our study are summarized as follows.

(i) There is no notable/significant difference in the properties of type II bursts and flares like start frequency, 


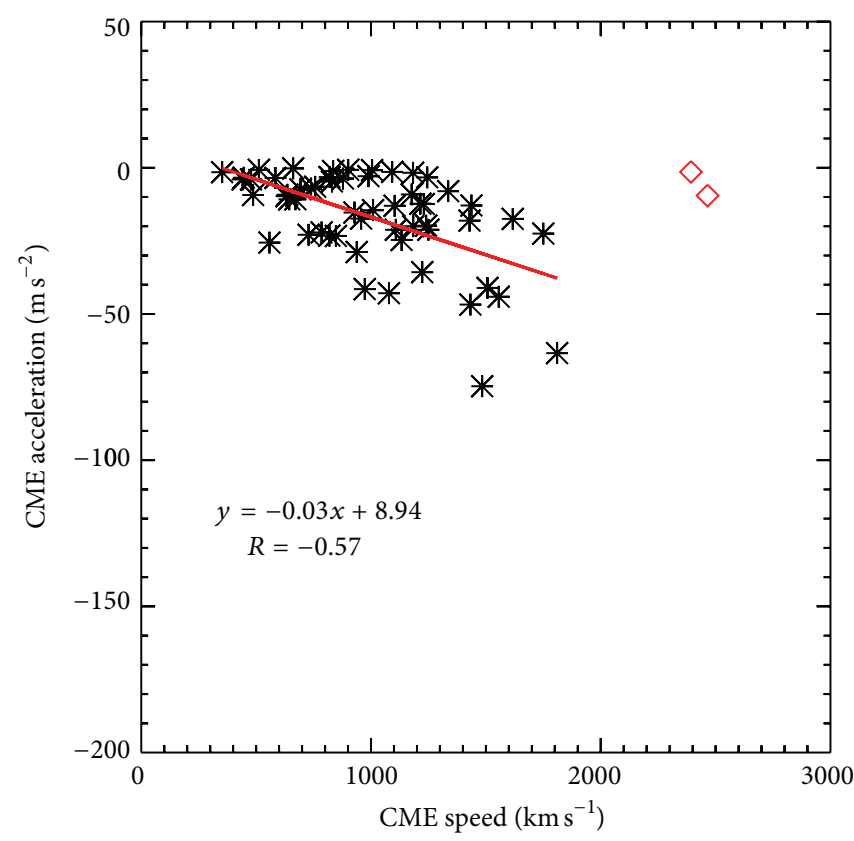

(a)

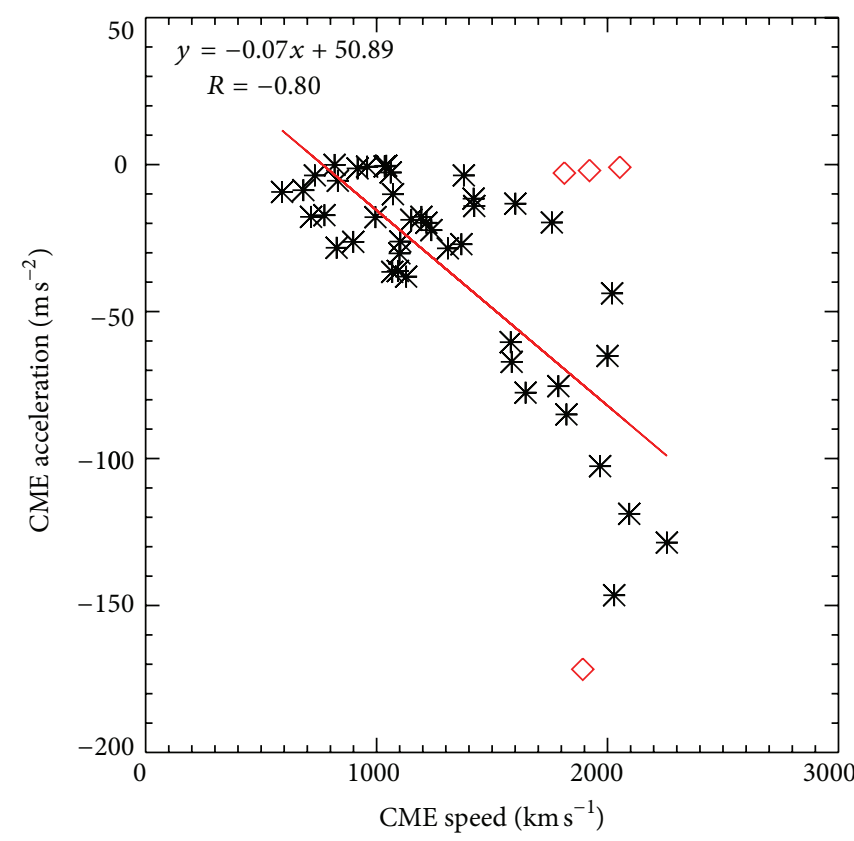

(b)

FIGURE 9: Correlation between speed and acceleration of CMEs for (a) rise phase events and (b) decay phase events (red diamonds are outlier points).

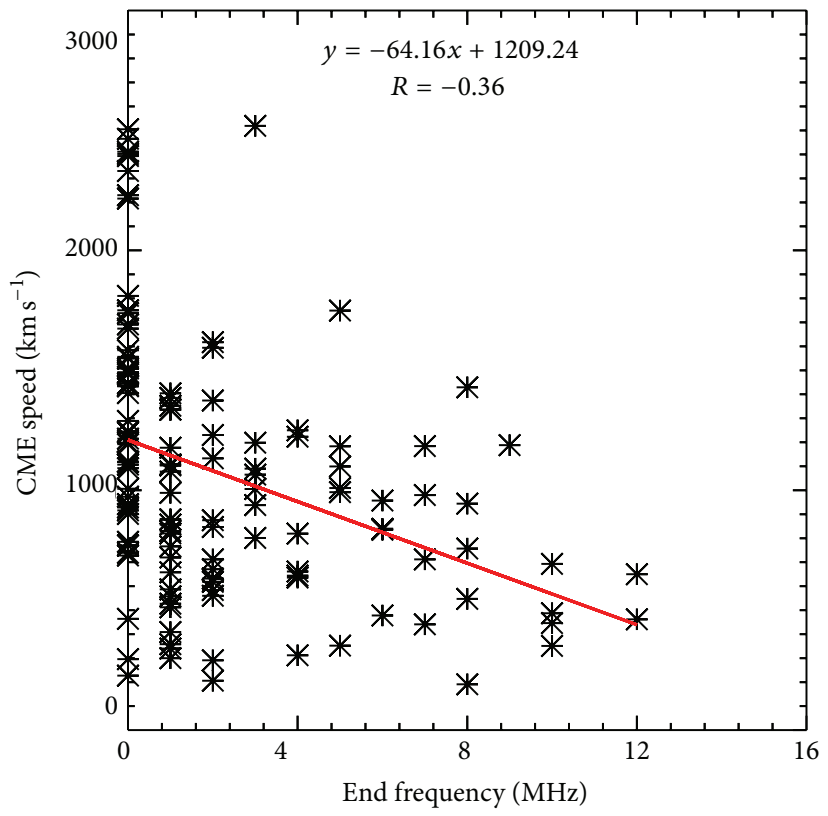

(a)

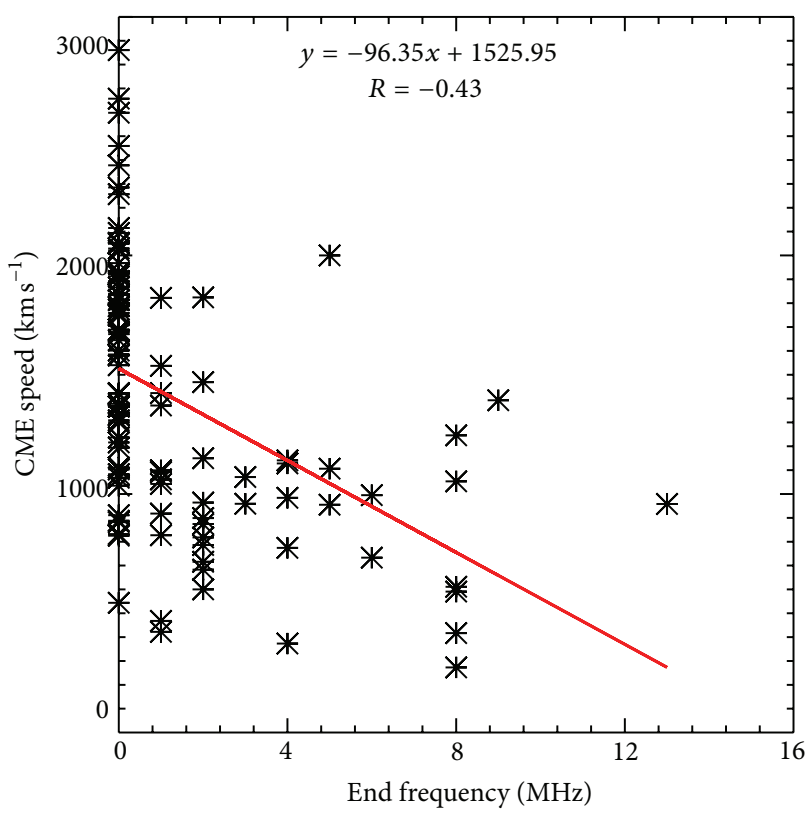

(b)

FIGURE 10: Correlation between end frequency and speed of CMEs for (a) rise phase events and (b) decay phase events.

end frequency, flare duration, rise time, and decay time in solar cycle rise phase events compared to solar cycle decay phase events, which indicates that these parameters of type II bursts do not depend on the phase of solar cycle.

(ii) The correlation between mean start frequency of type II bursts and drift rate is better for solar cycle decay phase events $(R=0.65)$ compared to solar cycle rise phase events $(R=0.50)$.

(iii) The mean CME speed of solar cycle decay phase events $\left(1373 \mathrm{~km} \mathrm{~s}^{-1}\right)$ is found to be slightly higher than the mean CME speed of solar cycle rise phase events $\left(1058 \mathrm{~km} \mathrm{~s}^{-1}\right)$. 
(iv) Only a small difference was found between the mean widths of solar cycle decay phase events $\left(276^{\circ}\right)$ compared to solar cycle rise phase events $\left(260^{\circ}\right)$.

(v) The mean acceleration in solar cycle decay phase events $\left(-15.18 \mathrm{~m} \mathrm{~s}^{-2}\right)$ is found to be higher than solar cycle rise phase events $\left(-1.32 \mathrm{~m} \mathrm{~s}^{-2}\right)$.

(vi) The shock speed of the DH-type II bursts in solar cycle decay phase events $\left(1260 \mathrm{~km} \mathrm{~s}^{-1}\right)$ is slightly larger than in solar cycle rise phase events $\left(1135 \mathrm{~km} \mathrm{~s}^{-1}\right)$.

(vii) The correlation between end frequency of the type II bursts and CME speed is very weak for both groups ( $R=0.43$ and $R=0.36$ for solar cycle rise phase and decay phase events, resp.).

(viii) There exists good correlation in solar cycle decay phase events between (i) CME speed and width $(R=0.79)$ and (ii) CME speed and acceleration $(R=-0.80)$, while the correlations are weak and nonexisting for solar cycle rise phase events with $R=$ 0.60 and $R=-0.57$.

About 229 DH-type II bursts associated with flares and CMEs are statistically studied in the 23rd solar cycle during the period 1997-2008. The number of events is larger for solar cycle rise phase $(127 / 232)$ as a maximum number of events are evolved during solar maximum and lower for solar cycle decay phase (102/229) as expected in a solar cycle. There is no notable/significant difference in the properties of type II bursts and flares like start frequency, end frequency, flare duration, rise time, and decay time in solar cycle rise phase events compared to solar cycle decay phase events. But the properties of CMEs (like CME speed and CME acceleration and correlation between (i) CME speed and width and (ii) CME speed and acceleration) associated with DH-type II bursts are better for solar cycle decay phase events compared to solar cycle rise phase events. Therefore, this clearly shows that more energetic CMEs are also produced during the solar cycle decay phase events and this may produce intense/severe geomagnetic storms at earth. Only the number of events follows the solar cycle, but more energetic CMEs may be produced at all time throughout the solar cycle and may produce stronger geomagnetic storms. The DH-type II bursts are driven by energetic CMEs [12]. Therefore the parameters of DH-type II bursts do not depend on the time of appearance (phase) in the solar cycle.

\section{Conflict of Interests}

The authors declare that there is no conflict of interests regarding the publication of this paper.

\section{Acknowledgments}

The authors thank the referee for his fruitful comments for the improvement of their paper. The authors greatly acknowledge the various online data centers of NOAA and NASA. Also, they thank the Wind/WAVES team for providing the type II catalog. The CME catalog used is provided by the
Center for Solar Physics and Space Weather, the Catholic University of America, in cooperation with Naval Research Laboratory and NASA.

\section{References}

[1] M. J. Reiner, "High-frequency Type II radio emissions associated with shocks driven by coronal mass ejections," Journal of Geophysical Research A: Space Physics, vol. 104, no. 8, pp. 1697916991, 1999.

[2] J. P. Wild and L. L. McCready, "Observations of the spectrum of high intensity solar radiations at metrewavelengths," Australian Journal of Scientific Research A, vol. 3, no. 4, pp. 541-557, 1950.

[3] R. Payne-Scott, D. E. Yabsley, and J. G. Bolton, "Relative times of arrival of bursts of solar noise on different radio frequencies," Nature, vol. 160, no. 4060, pp. 256-257, 1947.

[4] H. H. Malitson, J. Fainberg, and R. G. Stone, "Observation of a Type II solar radio burst to 37 Rsun,” Astrophysical Journal, vol. 14, pp. 111-114, 1973.

[5] A. Boischot, A. C. Riddle, J. B. Pearce, and J. W. Warwick, "Shock waves and Type II radiobursts in the interplanetary medium," Solar Physics, vol. 65, no. 2, pp. 397-404, 1980.

[6] H. V. Cane, N. R. Sheeley, and R. A. Howard, "Energetic interplanetary shocks, radio emission, and coronal mass ejections," Journal of Geophysical Research, vol. 92, no. 9, pp. 9869-9874, 1987.

[7] D. Lengyel-Frey and R. G. Stone, "Characteristics of interplanetary Type II radio emission and the relationship to shock and plasma properties," Journal of Geophysical Research, vol. 94, no. 1, pp. 159-167, 1989.

[8] J.-L. Bougeret, M. L. Kaiser, P. J. Kellogg et al., "WAVES: the radio and plasma wave investigation on the wind spacecraft," Space Science Reviews, vol. 71, no. 1-4, pp. 231-263, 1995.

[9] M. L. Kaiser, M. J. Reiner, N. Gopalswamy et al., “Type II radio emissions in the frequency range from $1-\mathrm{MHz}$ associated with the April 7, 1997 solar event," Geophysical Research Letters, vol. 25, no. 14, pp. 2501-2504, 1998.

[10] M. J. Reiner, M. L. Kaiser, J. Fainberg, J.-L. Bougeret, and R. G. Stone, "On the origin of radio emissions associated with the January 6-11, 1997, CME," Geophysical Research Letters, vol. 25, no. 14, pp. 2493-2496, 1998.

[11] V. Vasanth, S. Umapathy, B. Vršnak, and M. Anna Lakshmi, "Characteristics of Type-II radio bursts associated with flares and CMEs," Solar Physics, vol. 273, no. 1, pp. 143-162, 2011.

[12] N. Gopalswamy, S. Yashiro, M. L. Kaiser, R. A. Howard, and J.-L. Bougeret, "Characteristics of coronal mass ejections associated with long-wavelength Type II radio bursts," Journal of Geophysical Research A: Space Physics, vol. 106, no. 12, pp. 2921929229, 2001.

[13] B. Vršnak and E. W. Cliver, "Origin of coronal shock waves: iIInvited review," Solar Physics, vol. 253, no. 1-2, pp. 215-235, 2008.

[14] J. Magdalenić, B. Vršnak, S. Pohjolainen, M. Temmer, H. Aurass, and N. J. Lehtinen, "A flare-generated shock during a coronal mass ejection on 24 December 1996," Solar Physics, vol. 253, no. 1-2, pp. 305-317, 2008.

[15] A. Nindos, C. E. Alissandrakis, A. Hillaris, and P. PrekaPapadema, "On the relationship of shock waves to flares and coronal mass ejections," Astronomy and Astrophysics, vol. 531, article A31, 12 pages, 2011. 
[16] A. Lara, N. Gopalswamy, S. Nunes, G. Munoz, and S. Yashiro, "A statistical study of CMEs associated with metric Type II bursts," Geophysical Research Letters, vol. 30, no. 12, pp. SEP 4-1-SEP 44, 2003.

[17] N. Gopalswamy, A. Lara, R. P. Lepping, M. L. Kaiser, D. Berdichevsky, and O. C. St. Cyr, "Interplanetary acceleration of coronal mass ejections," Geophysical Research Letters, vol. 27, no. 2, pp. 145-148, 2000.

[18] V. Vasanth and S. Umapathy, "A statistical study on CMEs associated with DH-Type-II radio bursts based on their source location (limb and disk events)," Solar Physics, vol. 282, no. 1, pp. 239-247, 2013.

[19] V. Vasanth and S. Umapathy, "A statistical study on DH CMEs and its geo-effectiveness," ISRN Astronomy and Astrophysics, vol. 2013, Article ID 129426, 13 pages, 2013.

[20] N. Gopalswamy, S. Yashiro, G. Michalek et al., "The SOHO/LASCO CME catalog," Earth, Moon and Planets, vol. 104, no. 1-4, pp. 295-313, 2009.

[21] S. Yashiro, N. Gopalswamy, G. Michalek et al., "A catalog of white light coronal mass ejections observed by the $\mathrm{SOHO}$ spacecraft," Journal of Geophysical Research A: Space Physics, vol. 109, no. 7, article 105, 2004.

[22] G. E. Brueckner, J.-P. Delaboudiniere, R. A. Howard et al., "Geomagnetic storms caused by coronal mass ejections (CMEs): March 1996 through June 1997," Geophysical Research Letters, vol. 25, no. 15, pp. L3019-L3022, 1998.

[23] N. Gopalswamy, E. Aguilar-Rodriguez, S. Yashiro, S. Nunes, M. L. Kaiser, and R. A. Howard, "Type II radio bursts and energetic solar eruptions," Journal of Geophysical Research A: Space Physics, vol. 110, no. 12, article S07, 2005.

[24] N. Gopalswamy, "Coronal mass ejections and Type II bursts," in Solar Eruptions and Energetic Particles, N. Gopalswamy, R. Mewaldt, and J. Torsti, Eds., vol. 165 of Geophysical Monograph Series, p. 207, 2006.

[25] Y. Leblanc, G. A. Dulk, and J.-L. Bougeret, "Tracing the electron density from the corona to 1 AU," Solar Physics, vol. 183, no. 1, pp. 165-180, 1998.

[26] N. Gopalswamy, W. T. Thompson, J. M. Davila et al., "Relation between Type II bursts and CMEs inferred from STEREO observations," Solar Physics, vol. 259, no. 1-2, pp. 227-254, 2009.

[27] B. Vršnak, J. Magdalenić, and H. Aurass, "Comparative analysis of Type II bursts and of thermal and non-thermal flare signatures," Solar Physics, vol. 202, no. 2, pp. 319-335, 2001.

[28] B. Vršnak, "Processes and mechanisms governing the initiation and propagation of CMEs," Annales Geophysicae, vol. 26, no. 10, pp. 3089-3101, 2008.

[29] J. T. Gosling, E. Hildner, R. M. MacQueen, R. H. Munro, A. I. Poland, and C. L. Ross, "The speeds of coronal mass ejection events," Solar Physics, vol. 48, no. 2, pp. 389-397, 1976.

[30] Y.-J. Moon, G. S. Choe, H. Wang et al., "A statistical study of two classes of coronal mass ejections," Astrophysical Journal Letters, vol. 581, no. 1, pp. 694-702, 2002.

[31] B. Vršnak, "Deceleration of coronal mass ejections," Solar Physics, vol. 202, no. 1, pp. 173-189, 2001. 

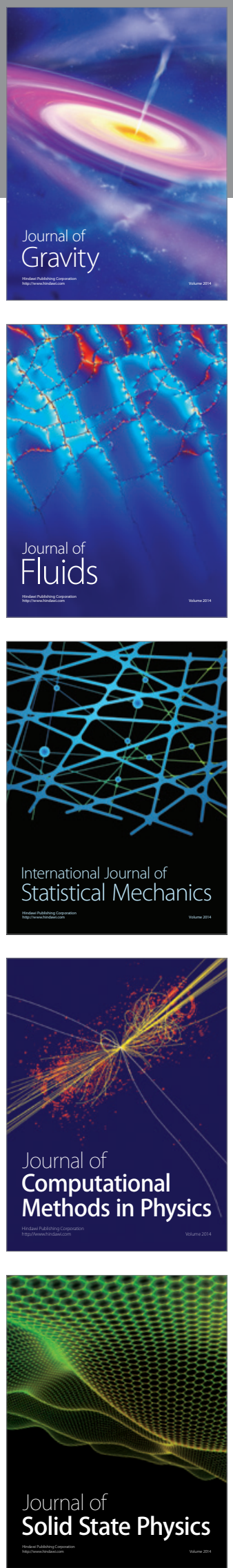

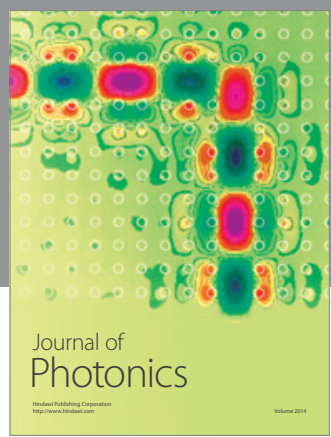

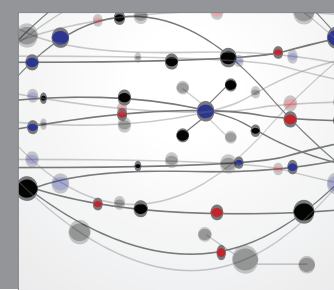

The Scientific World Journal

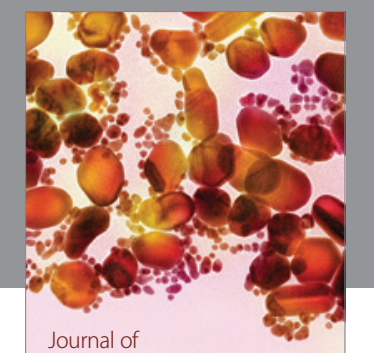

Soft Matter
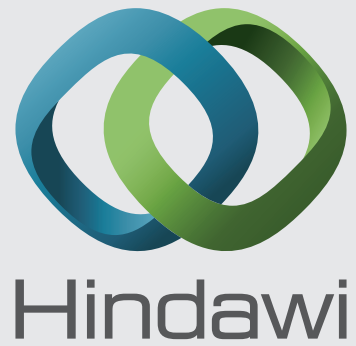

Submit your manuscripts at

http://www.hindawi.com
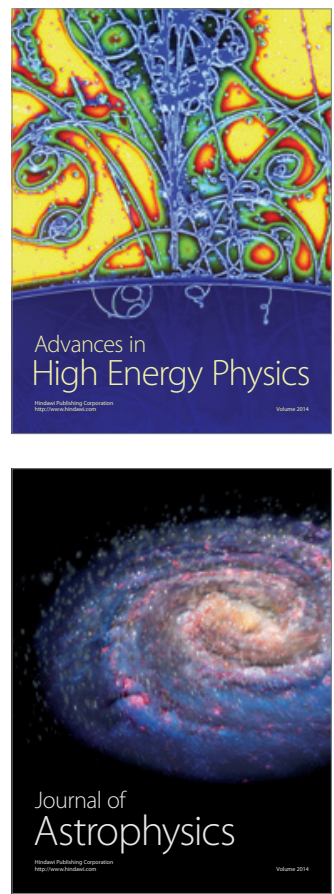
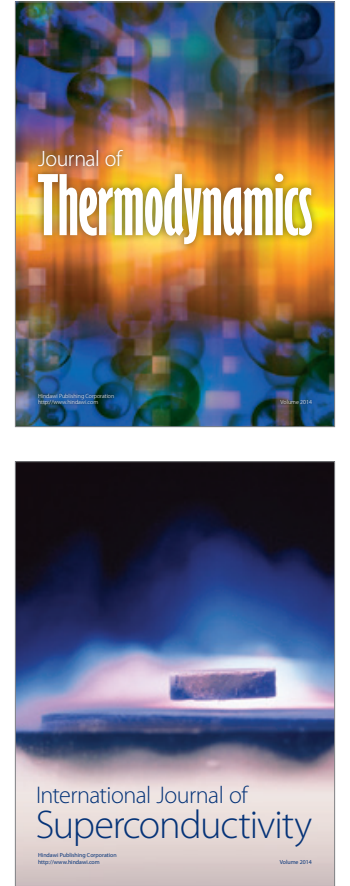
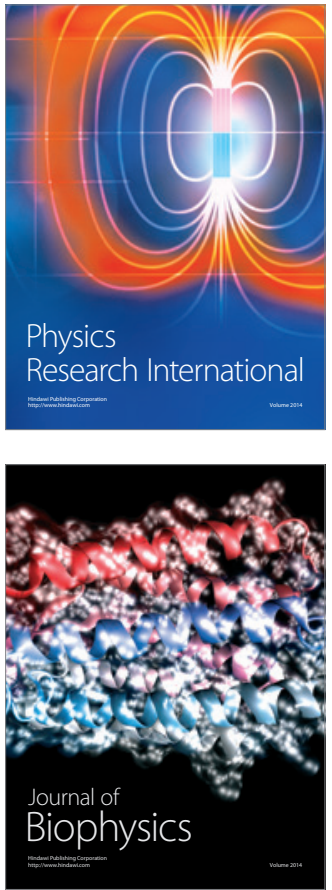
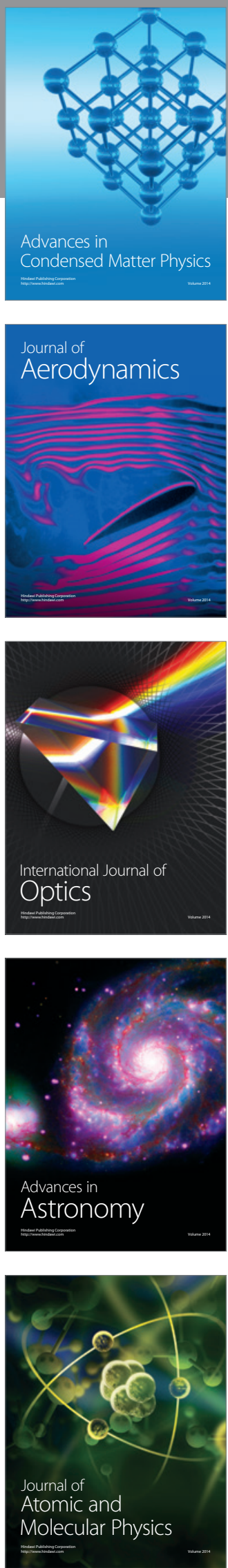